\title{
Interactions des protéines du lait et des oligoéléments
}

\author{
par \\ G. BRULÉ et J. FAUQUANT*
}

\section{Su $\mathrm{m} m$ a $r y$}

Repartition of trace elements ( $\mathrm{Cu}, \mathrm{Fe}, \mathrm{Zn}$, and $\mathrm{Mn}$ ) in milk between casein, whey proteins and non protein fraction was investigated. Casein bound about $95 \%$ of manganese and zinc and 50-75\% of copper and iron; 15 to $33 \%$ of copper and iron were present in non protein fraction and 18 to $33 \%$ of iron in whey proteins fraction.

Addition of trace elements in milk modified the repartition especially for copper and manganese. Affinity of cas $\alpha_{s i}$ and of cas $\beta$ for copper and iron compared to affinity for calcium and magnesium and competition between calcium and iron for chelation of casein suggested that phosphoserine groups are element - binding sites. Effect of orotical and citrate concentration on the repartition of trace element was studied. Citrate increased concentration of iron, manganese and copper in non protein fraction. Orotic acid only increased the copper content. The high degree of affinity of phosphoproteins. for trace element suggested to use phosphopeptides as chelators.

Key words

Milk - Trace element - Casein - Orotic acid - Citrate - Zinc - Copper Manganese - Iron - Calcium.

\footnotetext{
* Institut National de la Recherche Agronomique, Laboratoire de Recherches
} de Technologie Laitière, 65, rue de Saint-Brieuc - 35042 Rennes cedex. 


\section{INTRODUCTION}

L'absorption des oligoéléments en relation avec la composition de l'aliment dans lequel ils se trouvent a fait l'objet d'un certain nombre de travaux. Price et Bunce (1972) ainsi que Grecer et Snedeker (1980) ont étudié l'influence de la teneur en protéines, phosphore et calcium sur l'absorption du cuivre, manganèse et zinc; d'autres auteurs ont déterminé l'influence de ces facteurs sur l'absorption du fer (Monsen et Cook, 1979 ; Peters et al., (1971).

De l'ensemble de ces travaux, il est très difficile de conclure sur le rôle de tel ou tel constituant de l'aliment sur l'absorption des oligoéléments. Les contradictions apparentes dans les résultats des différents auteurs sont probablement dues aux connaissances insuffisantes des caractéristiques des aliments. Lorqu'ils ont étudié l'effet de la concentration en protéines, ils n'ont pas toujours pris en considération la nature des différentes protéines et leurs caractéristiques physico-chimiques, notamment leur affinité éventuelle avec les différents oligoéléments. Il en est de même de l'influence du phosphore qui doit dépendre du rapport des formes organiques et minérales et de la présence ou non d'alcalinoterreux tels que le magnésium ou calcium.

Il nous paraît donc important de connaître avant d'entreprendre de telles études la localisation des oligoéléments dans l'aliment, à savoir la nature des composés minéraux ou organiques qui les chélatent. C'est dans ce but que nous nous sommes proposés d'étudier la répartition de quelques oligoéléments dans le lait. 


\section{MATERIEL ET METHODE}

Les laits provenaient de deux fermes différentes et étaient prélevés au niveau du tank.

Le fractionnement des constituants du lait est réalisé par ultracentrifugation à $78000 \mathrm{~g}$ et par ultrafiltration sur cône (Centriflo CF 25).

Nous avons pu par détermination des taux en oligoéléments dans le lait écrémé, le surnageant de centrifugation et dans l'ultrafiltrat, établir leur répartition entre caséines, protéines solubles et fraction non protéique.

Le dosage des oligoéléments est réalisé par spectrophotométrie d'absorption atomique en four : $10 \mu \mathrm{l}$ d'échantillon étaient introduits dans le four, dilués ou non, déshydratés à $100^{\circ} \mathrm{C}$ pendant $60 \mathrm{~s}$, minéralisés à $450^{\circ} \mathrm{C}$ pendant $50 \mathrm{~s}$ et atomisés à $2200^{\circ} \mathrm{C}$ pendant $2 \mathrm{~s}$.

La concentration est déterminée par standardisation interne obtenue par ajout de l'oligoélément à deux cencentrations différentes.

\section{RESULTATS}

\section{Répartition des oligoéléments dans le lait}

La répartition du cuivre, fer, manganèse et zinc a été déterminée dans trois laits écrémés différents (tab. 1). La fraction caséinique complexe une quantité très importante des oligoéléments, à savoir plus de $95 \%$ pour le manganèse et le zinc et de 50 à $75 \%$ pour le cuivre et le fer. Les protéines non caséiniques ne présentent d'affinité que pour le fer. Les constituants non protéiques présents dans l'ultrafiltrat possèdent des propriétés chélatantes du cuivre et du fer puisqu'on y retrouve 15 à $33 \%$ de ces éléments.

La répartition de ces éléments dans le lait, lorsqu'ils y sont ajoutés à des concentrations de l'ordre de 5 et $10 \mathrm{ppm}$ est très différente de celle qui existe initialement (tab. 2). Nous constatons que la proportion de cuivre et de zinc non fixée au protéines est négligeable. La distribution du cuivre et zinc entre la fraction caséinique et les protéines solubles correspond approximativement au rapport des protéines contenues dans chacune de ces fractions.

\section{Affinité des caséines vis-à-vis du fer et du cuivre}

L'affinité de la caséine $\beta$ et caséine $\alpha_{\text {s1 }}$ vis-à-vis du cuivre et du fer est déterminée à l'aide de la représentation de Scatchard $\mathrm{R}$ $(-=\mathrm{Kn}-\mathrm{KR}) . \mathrm{R}$ est le nombre d'atomes d'oligoéléments associés 
TABLEAU 1 - TABLE 1

Répartition de quelques oligoéléments sur les fractions azotées de trois laits de mélange (ppb)

Repartition of some trace elements in three cow skimmilks ( $p p b)$

\begin{tabular}{|c|c|c|c|c|}
\hline & Lait écrémé & $\begin{array}{c}\text { Caséines } \\
\text { (centrifugat) }\end{array}$ & $\begin{array}{l}\text { Protéines solubles } \\
\quad \text { (surnageant) }\end{array}$ & $\begin{array}{l}\text { Azote non protéique } \\
\text { (perméat) }\end{array}$ \\
\hline $\mathrm{Cu}$ & $\begin{array}{r}96 \\
60 \\
100\end{array}$ & $\begin{array}{l}76 \\
40(66-76 \%) \\
76\end{array}$ & $\begin{array}{lll}4 & & \\
5 & (4-8 & \%) \\
8 & & \end{array}$ & $\begin{array}{l}16 \\
15 \\
15\end{array} \quad(15-25 \%)$ \\
\hline $\mathrm{Fe}$ & $\begin{array}{l}170 \\
105 \\
180\end{array}$ & $\begin{array}{r}110 \\
65 \\
90\end{array}$ & $\begin{array}{l}30 \\
35 \\
60\end{array} \quad(18-33 \%)$ & $\begin{array}{l}30 \\
15 \\
30\end{array} \quad(15-33 \%)$ \\
\hline $\mathrm{Mn}$ & $\begin{array}{l}80 \\
86 \\
54\end{array}$ & $\begin{array}{l}76 \\
82(95 \%) \\
51\end{array}$ & $\begin{array}{l}1,0 \\
1,5 \quad(1-2 \%) \\
2,0\end{array}$ & $\begin{array}{l}2,9 \\
3,5(4 \%) \\
2,2\end{array}$ \\
\hline $\mathrm{Zn}$ & $\begin{array}{l}5000 \\
5400 \\
5600\end{array}$ & $\begin{array}{l}4880 \\
5280(97 \%) \\
5450\end{array}$ & 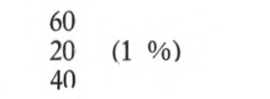 & $\begin{array}{r}60 \\
100 \\
110\end{array} \quad$ (2 \%) \\
\hline
\end{tabular}




\section{TABLEAU $2-T A B L E 2$}

Répartition d'oligoéléments ajoutés au lait (ppm) -

Reparttion of added trace element in skimmilks (ppm)

\begin{tabular}{|c|c|c|c|c|}
\hline \multicolumn{2}{|c|}{ Lait écrémé } & \multirow{2}{*}{$\begin{array}{l}\text { Caséines } \\
\begin{array}{l}4.40(83 \%) \\
7,80(82 \%)\end{array}\end{array}$} & Protéines solubles & \multirow{2}{*}{$\begin{array}{l}\text { Fraction } \\
\text { non protéique } \\
0,24 \quad(2 \%)\end{array}$} \\
\hline $\mathrm{Cu}$ & $\begin{array}{r}5,00 \\
10,00\end{array}$ & & 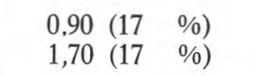 & \\
\hline $\mathrm{Fe}$ & $\begin{array}{r}6 \\
12\end{array}$ & $\begin{array}{l}3.10(60 \%) \\
6,40(57 \%)\end{array}$ & $\begin{array}{lll}1,10 & (21,5 \%) \\
2,90 & (25 & \%)\end{array}$ & 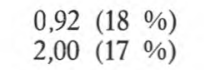 \\
\hline $\mathrm{Mn}$ & $\begin{array}{l}4,7 \\
9,5\end{array}$ & $\begin{array}{ll}3,38 & (72 \%) \\
6,65 & (74 \%)\end{array}$ & 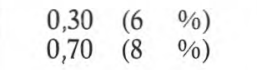 & $\begin{array}{l}1,02(21 \%) \\
1,57 \quad(18 \%)\end{array}$ \\
\hline $\mathrm{Zn}$ & $\begin{array}{r}9,6 \\
15,5\end{array}$ & $\begin{array}{l}6.89(83 \%) \\
9,69(78 \%)\end{array}$ & 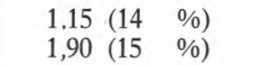 & 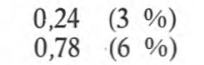 \\
\hline
\end{tabular}

par mole de protéines, $\mathrm{n}$ le nombre de sites de fixation et A la concentration de l'oligoélément à l'état libre. L'affinité des caséines vis-à-vis des oligoéléments est beaucoup plus forte que vis-à-vis du calcium et magnésium (fig. 1 et 2 ). Le nombre de sites de fixation est très

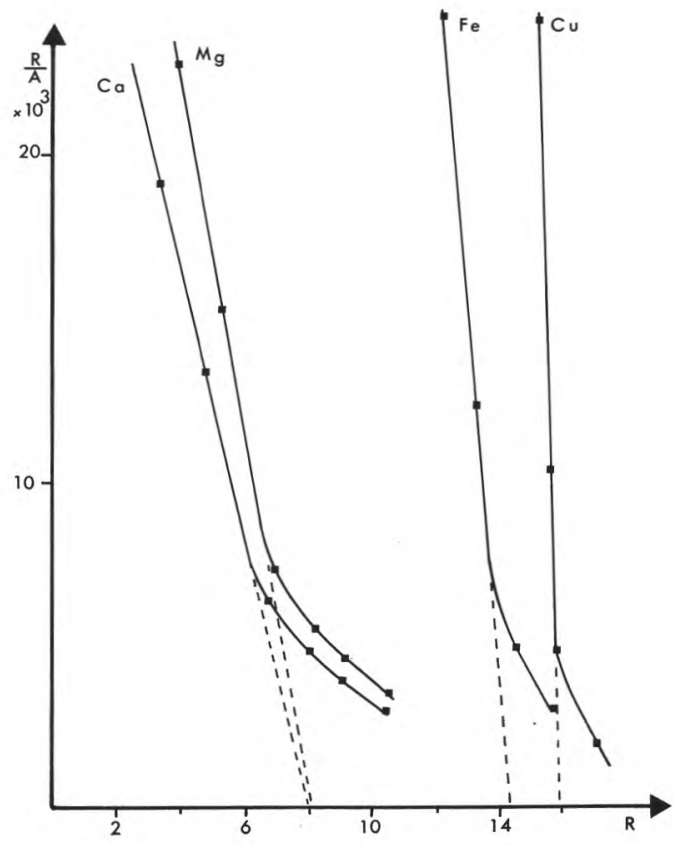

fig. 1

Affinité de la cas. $\alpha_{\mathbb{S}_{1}}$ vis-à-vis du calcium, magnésium, fer et cuivre. (Représentation de SCATCHARD).

Affinity of Cas.a for calcium, magnesium, iron and copper (SCATCHARD). 

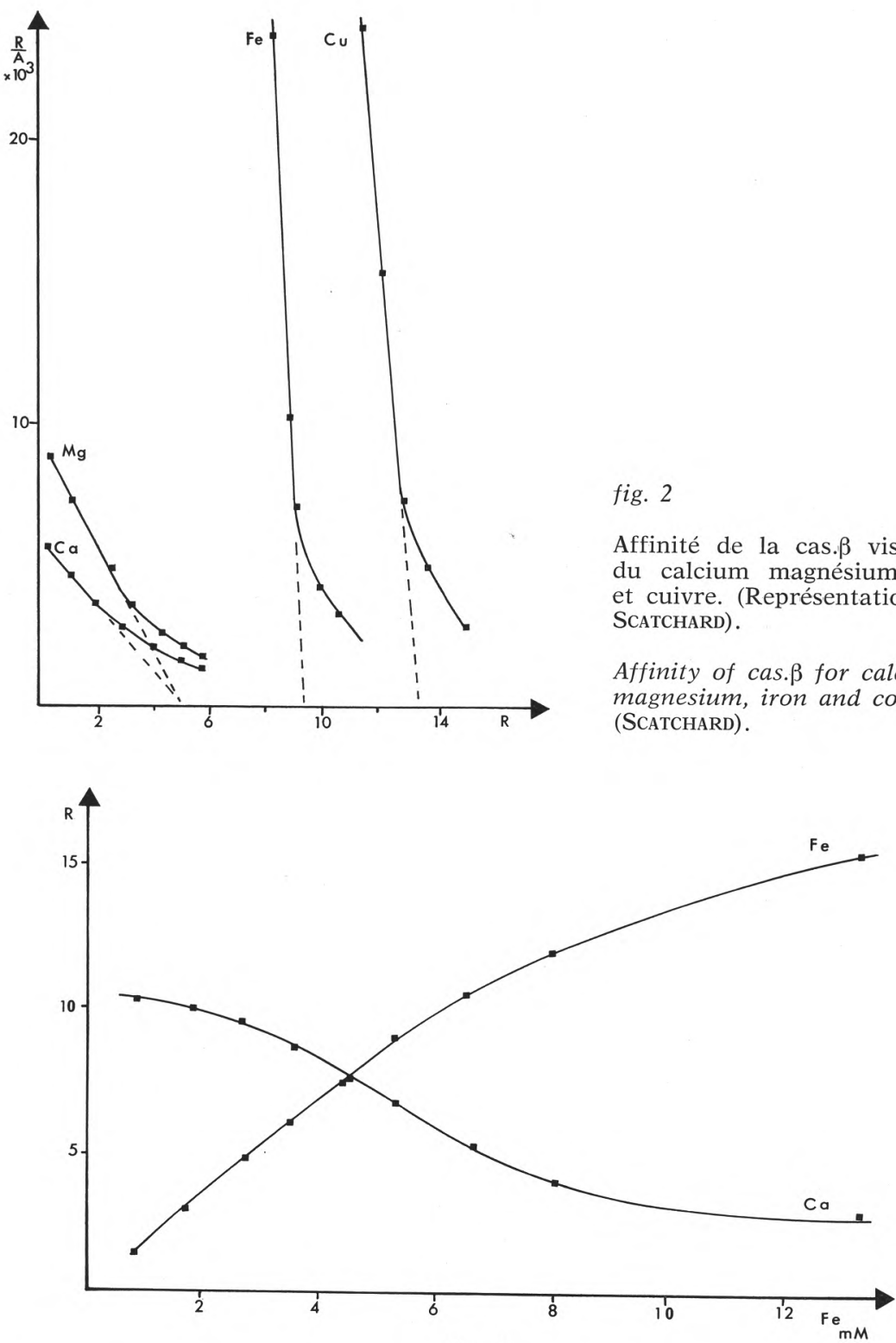

fig. 3 fig. 2

Affinité de la cas. $\beta$ vis-à-vis du calcium magnésium, fer et cuivre. (Représentation de SCATCHARD).

Affinity of cas. $\beta$ for calcium, magnesium, iron and copper. (SCATCHARD). 
élevé puisque selon ces représentations la caséine $\beta$ peut chélater 9 atomes de fer et 13 atomes de cuivre et la caséine $\alpha$ si 14 atomes de fer et 16 atomes de cuivre. Le plus grand pouvoir chélatant de la caséine $\alpha_{\text {si }}$ par rapport à celui de la caséine $\beta$ est probablement dû à son taux de phosphorylation plus élevé. En effet, nous avons montré sur la caséine $\alpha$ si qu'il y a compétition entre le fer et le calcium au niveau des sites de fixation phosphosériques. La figure 3 met bien en évidence l'évolution du calcium et du fer liés à la caséine $\alpha$ sis lors de l'addition de fer à une solution de caésinate de calcium. La fixation de 15 atomes de fer par molécule de caséine $\alpha_{1}$ se traduit par une solubilisation d'environ 7 atomes de calcium lesquels se trouvaient préférentiellement fixés sur les sites phosphosériques.

\section{Influence des sequestrants non protéiques sur la répartition des oligoéléments}

Nous avons examiné l'influence du citrate et de l'acide orotique sur la répartition du cuivre, fer et manganèse. Les teneurs en éléments ultrafiltrables des laits auxquels ont été ajoutés $15 \mathrm{ppm}$ de fer et $25 \mathrm{ppm}$ de cuivre et de zinc sont présentés dans le tableau 3 ; y figurent également les variations obtenues après addition de 100 ou $200 \mathrm{mg}$ d'acide orotique et de $500 \mathrm{ou} 1000 \mathrm{mg}$ d'acide citrique. L'acide orotique a un pouvoir sequestrant du cuivre puisqu'il augmente d'environ $50 \%$ la teneur en élément soluble, alors qu'il n'a pas d'effet sur les autres éléments étudiés. L'addition de citrate augmente d'environ $100 \%$ les teneurs en zinc et fer solubles alors qu'elle n'aug-

\section{TABEAU 3 - TABLE 3}

Influence de l'acide orotique et citrique sur la répartition d'oligoéléments ajoutés (ppm)

Effect of orotic acid and citrate on repartition of added trace elements ( $p$ pm)

\begin{tabular}{|c|c|c|c|c|c|c|}
\hline & & \multirow{3}{*}{$\begin{array}{c}\text { Teneur en élément } \\
\text { soluble } \\
\text { dans le témoin }\end{array}$} & \multicolumn{4}{|c|}{$\begin{array}{l}\text { Variations des teneurs } \\
\text { en éléments solubles }\end{array}$} \\
\hline & & & \multicolumn{2}{|c|}{ Acide orotique } & \multicolumn{2}{|c|}{ Acide citrique } \\
\hline & & & $100 \mathrm{mg}$ & $200 \mathrm{mg}$ & $500 \mathrm{mg}$ & $1000 \mathrm{mg}$ \\
\hline $\mathrm{Cu}$ & $(26 \mathrm{ppm})$ & $0,80-1,10$ & $+0,40$ & $+0,55$ & $+0,20$ & $+0,30$ \\
\hline $\mathrm{Fe}^{2+}$ & (15 ppm) & $1,25-1,55$ & NS & NS & $+1,40$ & $+2,00$ \\
\hline $\mathrm{Zn}$ & (25 ppm) & $0,55-0,60$ & $-0,10$ & $-0,05$ & $+0,50$ & $+0,75$ \\
\hline
\end{tabular}


mente que de $20 \%$ les teneurs en cuivre soluble. En doublant les teneurs en acide orotique et citrate du lait, on double les teneurs en cuivre, fer et zinc présents dans la phase soluble du lait.

\section{CONCLUSION}

L'étude de la répartition des oligoéléments présents dans le lait met en évidence la grande affinité des caséines pour le zinc et le manganèse, celle des protéines solubles (lactoferrine) pour le fer et la présence de sequestrants non protéiques du fer et cuivre. Le pouvoir chélatant de la lactoferrine et des constituants non protéiques est limité ce qui explique que dans les laits complémentés en oligoéléments, une proportion très forte de ces éléments se trouve fixée sur les caséines. Il semble que le nombre de sites de fixation dépend au moins partiellement du taux de phosphorylation des caséines, ce qui est en accord avec les résultats de DemotT et Dincer (1976) qui ont étudié la distribution du fer entre les différentes caséines (Cas $\alpha_{\mathrm{si}_{1}}>$ Cas $\beta>$ Cas K).

Ces résultats nous ont conduits à étudier le pouvoir sequestrant des phosphopeptides que nous avons isolés des hydrolysats enzymatiques de caséine (Brulé et al., 1980. Brulé et al., 1980). Ces peptides ont la possibilité de chélater des quantités d'oligoéléments pouvant atteindre $10 \%$ de leur poids sans en altérer la solubilité.

L'ensemble de ces résultats montre l'importance des phosphoprotéines dans la séquestration des oligoéléments. Reste à savoir si l'excellent pouvoir chélatant de ces protéines et éventuellement des phosphopeptides est un élément favorable à l'absorption des minéraux. Des travaux réalisés par Mykkanen et Wasserman (1980) sur l'absorption du calcium et ceux de Mathan et al. (1979) sur l'absorption du fer semblent le confirmer.

\section{Bibliographie}

Brulé (G.), Roger (L.), Fauquant (J.) et Piot (M.) (1980). - Procédé de traitement d'une matière première à base de caséine, contenant des phosphocaséinates de cations bivalents, produits obtenus et applications. Brevet $\mathrm{n}^{\circ} 8002280$,

Brulé (G.), Roger (L.), Fauquant (J.) et Piot (M.) (1980). - Procédé de traitement d'une matière première à base de caséine contenant des phosphocaséinates de cations monovalents ou leurs dérivés, produits obtenus et applications. Brevet $\mathrm{n}^{\circ} 8002281$.

Demott (B. J.) and Dincer (B.) (1976), - Binding added iron to various milk proteins. J. Dairy Sc., 59 (9), 1557-1559.

GRECER (J. L.) and SNEDEKer (S. M.) (1980). - Effect of dietary protein and phosphorus level on the utilization of zinc, copper and manganese by adult males. J. Nutr., 110, 2243-2253. 
Mathan (V. I.), Baker (S. J.), Sood (S. K), Ramachan-Dran (K.) and Ramalingaswami (V.) (1979). - W.H.O. sponsored collaborative studies on nutritional anaemia in India. Br. J. Nutr., 42, 391.

Monsen (E. R.) and CooK (J.D.) (1979). - Food iron absorption in human subjects V effects of the major dietary constituents of a semisynthetic meal. Am. J. Clin. nutr., 32, 804-808.

MYKKanen (H.M.) and Wasserman (R. H.) (1980). - Enhanced absorption of calcium by casein phosphopeptides in rachidic and normal chicks. J. Nutr., 110, 2141-2148.

Peters (T.) Jr., Apt (L.) and Ross (J. R.) (1971). - Effect of phosphates upon iron absorption studied in normal human subjects and in an experimental model using dialysis. Gastroenterology, 61, 315-322.

Price (N. O.) and Bruce (G. E.) (1972). - Effect of nitrogen and calcium on balance of copper manganese and zinc in pre adolescent girls. Nutr. Rep. Int., 5, 275-280. 